\title{
Spectral Estimation of Digital Signals by the Orthogonal Kravchenko Wavelets $\left\{\widetilde{h_{a}(t)}\right\}$
}

\author{
Victor Kravchenko ${ }^{1}$, Hector Perez Meana ${ }^{2}$, Volodymyr Ponomaryov ${ }^{2}$, \\ and Dmitry Churikov ${ }^{1}$ \\ ${ }^{1}$ Kotel'nikov Institute of Radio Engineering and Electronics of RAS, \\ Mokhovaya 11, build. 7, 125009, Moscow, Russia \\ olegk@lianet.ru, mpio_nice@mail.ru \\ ${ }^{2}$ National Polytechnic Institute of Mexico, AV. SANTA ANA No.1000, \\ Col. San Francisco Culhuacan 04430, Mexico-city, Mexico \\ hmperezm@ipn.mx, vponomar@ipn.mx
}

\begin{abstract}
In this article, the approach based on the orthogonal Kravchenko wavelets $\left\{\widetilde{h_{a}(t)}\right\}$ is proposed. There is shown that obtained structures have some advantages in comparison with spectral wave analysis of ultra wideband (UWB) signals that are widely used in the remote sensing. This approach based on application of wavelets as spectral kernels is considered in the problems of digital UWB signal processing. In communication theory, the signals are represented in the form of linear combination of elementary functions. Application of spectral analysis of UWB signals in basis of digital functions in comparison with the spectral harmonious analysis gives certain advantages which are defined by the physical nature of the signal representation. Optimal processing in spectral area in comparison with the time possesses has advantages on application of numerical algorithms. The physical characteristics and analysis of numerical experiment confirm efficiency of new wavelets in the spectral estimation and digital UWB signal processing.
\end{abstract}

Keywords: Atomic functions, Wavelets, Remote sensing, Digital ultra wideband signal processing.

\section{Introduction}

Application of the spectral analysis of signals in basis of digital functions in comparison with the spectral harmonious offers certain advantages which are defined by the physical nature of representation of the signals [1-11] in the remote sensing problems $[1,2]$. Signals can be set in the form of some linear combination of elementary functions [3-5]

$$
s(t)=\sum_{k=0}^{N-1} C(k) \varphi(k, t),
$$

where $\varphi(k, t)$ is an elementary function of number $k$, and $N$ is quantity of the functions used in the decomposition. At approximation the generalized Fourier transformation of a kind 


$$
C(k)=\int_{0}^{T} s(t) \varphi(k, t) d t
$$

provides the minimum value of mean-square error. Thus, there is signal decomposition on some basis, which in many problems of digital signal processing can not be orthogonal. The work purpose is consisted of justify the advantages of the new Kravchenko wavelets.

\section{Atomic Functions $h_{a}(t)$ and Their Properties}

Atomic functions [5-8], [12-17] $h_{a}(t)(a>1)$ are finite decisions of the functionaldifferential equation

$$
y^{\prime}(t)=\frac{a^{2}}{2}(y(a t+1)-y(a t-1)),
$$

where $a$ is any real number. The basic properties of $h_{a}(t)$ are the following.

$1^{\mathrm{O}}$. Compact support: $\left[-\frac{1}{a-1} ; \frac{1}{a-1}\right] . \quad 2^{\mathrm{O}} . h_{a}(t)=\frac{a}{2}$ for $t \in\left[-\frac{a-2}{a(a-1)} ; \frac{a-2}{a(a-1)}\right]$, $a \geq 2$. $\quad 3^{\mathrm{O}}$. Position of inflection $t=\left[\mp \frac{1}{a}, \frac{a}{4}\right] \cdot 4^{\mathrm{O}}$. The Fourier transform of $h_{a}(t)$ looks like

$$
\hat{h}_{a}(\omega)=\prod_{k=1}^{\infty} \operatorname{sinc}\left(\frac{\omega}{a^{k}}\right) .
$$

In practical calculations, it is enough to limit the product (4) to a small number of terms, as they quickly aspire to unit with growth of $k$.

$5^{\mathrm{O}}$. Derivatives of $h_{a}(t)$ are expressed through shifts-compression of the function recurrently by means of a parity (3).

\section{Constructing of the Orthogonal Kravchenko Wavelets}

The orthogonal Kravchenko wavelets that is based on AF $h_{a}(t)$ and have smooth Fourier transform are proposed. It allows providing the best time localization in comparison with Kotelnikov-Shannon wavelet. Their construction [7-11] is carried out by means of the quadrature mirror filters $\widehat{m}_{0}(\omega)$. For maintenance of orthogonality performance, the following conditions in transitive area are necessary

$$
\left|\widehat{m}_{0}(\omega)\right|^{2}+\left|\hat{m}_{0}(\omega-2 \pi)\right|^{2}=2 .
$$


The Fourier transform of scaling function [7-11] is defined from the equation

$$
\hat{\varphi}(\omega)=\frac{1}{\sqrt{2}} \hat{m}_{0}\left(\frac{\omega}{2}\right) \hat{\varphi}\left(\frac{\omega}{2}\right) \Leftrightarrow \hat{\varphi}(\omega)=\prod_{k=1}^{\infty} \frac{1}{\sqrt{2}} \hat{m}_{0}\left(\frac{\omega}{2^{k}}\right) .
$$

The Fourier transform of wavelet function can be written as

$$
\hat{\psi}(\omega)=\frac{1}{\sqrt{2}} \hat{g}\left(\frac{\omega}{2}\right) \hat{\varphi}\left(\frac{\omega}{2}\right)
$$

where $\hat{g}(\omega)=\mathrm{e}^{-i \omega} \hat{m}_{0}(\omega+\pi)$. Considering the properties of $h_{a}(t)$ it is possible to simplify a construction of function $\widehat{m}_{0}(\omega)$. For this purpose, we modify $h_{a}(t)$ according to following additional conditions.

$6^{\mathrm{O}}$. The support of function $t \in\left[-\frac{2}{3} \pi ; \frac{2}{3} \pi\right] \cdot 7^{\mathrm{O}} . \tilde{h}_{a}(t)=\sqrt{2}$ for $t \in\left[-\frac{1}{3} \pi ; \frac{1}{3} \pi\right]$.

$8^{\mathrm{O}}$. For interface of the Fourier transforms $\tilde{h}_{a}\left(\frac{1}{2} \pi\right)=1$.

Thus, as $\hat{m}_{0}(\omega)$ we take $\tilde{h}_{a}(t)$ with formal replacement of argument $t=\omega$. Properties $1^{\text {st }}$ and $7^{\text {th }}$ allow rewriting (7) in such a form

$$
\hat{\varphi}(\omega)=\tilde{h}_{a}\left(\frac{\omega}{2}\right)
$$

so long as $\tilde{h}_{a}\left(\frac{\omega}{2}\right) \neq 0$ only for $t \in\left[-\frac{4}{3} \pi ; \frac{4}{3} \pi\right]$, where $\tilde{h}_{a}\left(\frac{\omega}{4}\right)=\sqrt{2}$. From $5^{\text {th }}$ property, it follows that in transitive area $h_{a}(t)$ is symmetric relatively of inflection. Therefore, if as function $\tilde{h}_{a}(\omega)$ we take a square root of the $h_{a}(t)$, then the condition (4) is carried out. Hence, the function $\tilde{h}_{a}(\omega)$ is constructed as follows:

(a) replacement of variable $t=\frac{3}{2 \pi} \omega$, (b) to satisfy the property $6^{\text {th }}$ it should be $a \geq 4$, (c) scaling of function and argument, and also taking a square root.

Thus, we obtain the function for the scaling equation with various velocities of increase and recession depending of parameter $a$

$$
\tilde{h}_{a}(\omega)=\frac{2}{\sqrt{a}} \sqrt{h_{a}\left(\frac{2}{a \pi} \omega\right)} .
$$

Finally, we can write the Fourier transforms for scaling and wavelet functions as follows:

$$
\hat{\varphi}(\omega)=\frac{2}{\sqrt{a}} \sqrt{h_{a}\left(\frac{\omega}{a \pi}\right)}, \hat{\psi}(\omega)=\mathrm{e}^{-i \omega} \frac{2 \sqrt{2}}{a} \sqrt{h_{a}\left(\frac{2}{a \pi}(\omega+\pi)\right) h_{a}\left(\frac{\omega}{a \pi}\right)} .
$$



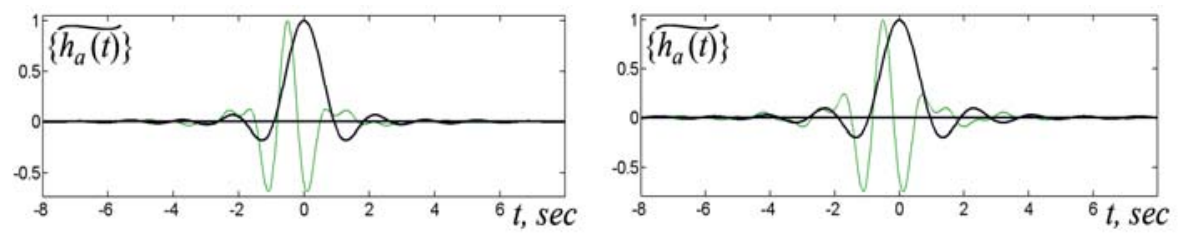

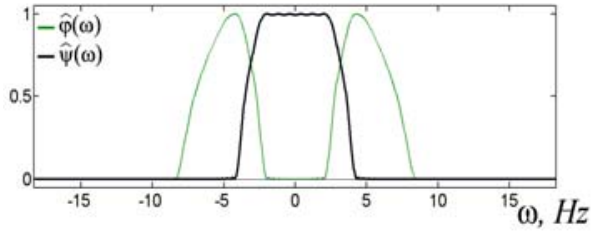

a)

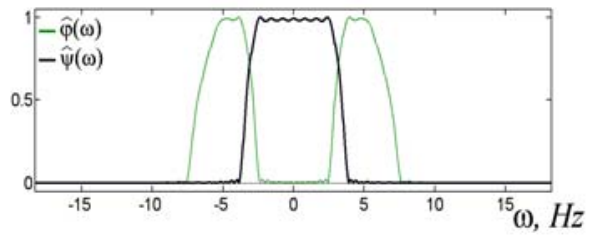

b)

Fig. 1. The Kravchenko $\left\{\widetilde{h_{a}(t)}\right\}$ scaling (dark line) and wavelet (light line) functions for $a=4$ (a) and $a=6(b)$

As an example, the Kravchenko wavelets $\left\{\widetilde{h_{a}(t)}\right\}$ and their spectrums are exposed in Fig. 1 for $a=4,6$.

Energy of scaling and wavelet functions on $N^{\text {th }}$ level is equal to energy of scaling function of $(N+1)^{\text {th }}$ level because

$$
|\hat{\varphi}(\omega)|^{2}+|\hat{\psi}(\omega)|^{2}=\left|\hat{m}_{0}\left(\frac{\omega}{4}\right)\right|^{2}=\left|\hat{\varphi}\left(\frac{\omega}{2}\right)\right|^{2} .
$$

Constructed functions are satisfying with all wavelet properties [6-10] and also they are orthogonal ones.

\section{Physical Characteristics}

To study the scaling and wavelet functions, we use the modified physical characteristics [5]: wideband index $\mu$; central frequency of spectral density function (SDF) $f_{0}$; relative position of SDF maximum (defined as $\gamma_{1}=f_{m} / f_{0}$, where $f_{m}$ is frequency of the main maximum); relative position of $S D F$ first zero $\gamma_{2}$; relative $S D F$ width on level -3 $d B \quad \gamma_{3}$; relative SDF width on level $-6 d B \quad \gamma_{4}$; coherent amplification defined as $\gamma_{7}=\frac{1}{\tau} \int_{-\tau / 2}^{\tau / 2}|\varphi(t)| d t$; equivalent noise band $\gamma_{8} ;$ maximum level of sidelobes (in $\left.\mathrm{dB}\right)$ $\gamma_{9}$. Mentioned physical characteristics of the Kravchenko wavelets and known ones for comparison are exposed in Table 1 below. 
Table 1. Physical characteristics of the Kravchenko wavelets in comparison with known ones

\begin{tabular}{|c|c|c|c|c|c|c|c|c|}
\hline & $a$ & $\gamma_{1}$ & $\gamma_{2}$ & $\gamma_{3}$ & $\gamma_{4}$ & $\gamma_{7}$ & $\gamma_{8}$ & $\gamma_{9}$ \\
\hline \multicolumn{9}{|c|}{ Kravchenko wavelets $\left\{\widetilde{h_{a}(t)}\right\}$} \\
\hline$\varphi(t)$ & \multirow{2}{*}{4} & 0 & 0,676 & 0,563 & 0,608 & 0,704 & 4,158 & $-19,65$ \\
\hline$\psi(t)$ & & 0,850 & 0,665 & 0,688 & 0,836 & 0,810 & 4,032 & $-19,77$ \\
\hline$\varphi(t)$ & \multirow{2}{*}{5} & 0 & 0,637 & 0,546 & 0,585 & 0,655 & 3,744 & $-17,36$ \\
\hline$\psi(t)$ & & 0,874 & 0,577 & 0,642 & 0,762 & 0,713 & 3,442 & $-17,30$ \\
\hline$\varphi(t)$ & \multirow{2}{*}{6} & 0 & 0,619 & 0,534 & 0,568 & 0,629 & 3,505 & $-16,73$ \\
\hline$\psi(t)$ & & 0,876 & 0,528 & 0,614 & 0,710 & 0,671 & 3,230 & $-16,55$ \\
\hline \multicolumn{9}{|c|}{ Kotelnikov-Shannon wavelet } \\
\hline$\varphi(t)$ & - & 0 & 0,540 & 0,494 & 0,512 & 0.500 & 2,429 & $-11,73$ \\
\hline$\psi(t)$ & - & 0,996 & 0,290 & 0,489 & 0,523 & 0,458 & 2,037 & $-10,56$ \\
\hline \multicolumn{9}{|c|}{ Meyer wavelet } \\
\hline$\varphi(t)$ & 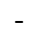 & 0 & 0,654 & 0,523 & 0,557 & 0,586 & 3,430 & $-29,11$ \\
\hline$\psi(t)$ & - & 0,863 & 0,648 & 0,574 & 0,671 & 0,565 & 3,196 & $-27,13$ \\
\hline \multicolumn{9}{|c|}{ Daubechies 4 wavelet } \\
\hline$\varphi(t)$ & - & 0 & 0,500 & 0,284 & 0,329 & 0,352 & 2,485 & $-10,92$ \\
\hline$\psi(t)$ & - & 0,847 & 0,637 & 0,375 & 0,506 & 0,343 & 2,362 & $-10,63$ \\
\hline
\end{tabular}

\section{Models of Ultra Wideband Signals}

We consider of following models of ultra wideband (UWB) signals [3, 4], [5-7] in respect of remote sensing problems $[1,2]$ :

1. $y_{1}(t)=-\left(H\left(\frac{t+0,5}{\tau}\right)-H\left(\frac{t-0,5}{\tau}\right)\right) \cdot \operatorname{sgn}(t)$,

2. $y_{2}(t)=(-1)^{n} \cdot \sin \left(\pi n \frac{t}{\tau}\right) \cdot \exp \left(-\left|\frac{t}{\tau}\right|\right) \cdot\left(H\left(\frac{t}{\tau}+1\right)-H\left(\frac{t}{\tau}-1\right)\right)$,

3. $y_{3}(t)=-\frac{2 t}{\tau^{2}} \cdot \exp \left(-\left(\frac{t}{\tau}\right)^{2}\right), \quad$ 4. $y_{4}(t)=-\frac{2}{\tau^{2}}\left(1-\frac{2 t^{2}}{\tau^{2}}\right) \cdot \exp \left(-\left(\frac{t}{\tau}\right)^{2}\right)$

5. $y_{5}(t)=\exp \left(-\left(\frac{t}{2 \tau}\right)^{2}\right) n ! \sum_{k=0}^{[n / 2]}\left(-\frac{1}{2}\right)^{k} \frac{(t / \tau)^{n-2 k}}{(n-2 k) ! k !}$, 
where $H(t)=\left\{\begin{array}{ll}0, & t<0, \\ 1, & t \geq 0 .\end{array}\right.$ is the Heaviside function and $\operatorname{sgn}(t)$ is the sign function. Their physical characteristics are presented in Table 2.

Table 2. Model UWB signals and their physical characteristics

\begin{tabular}{llrrrrrrrrrr}
\hline No. & \multicolumn{1}{c}{$\begin{array}{c}\text { Model UWB } \\
\text { signals }\end{array}$} & $\mu$ & $\gamma_{1}$ & $\gamma_{2}$ & $\gamma_{3}$ & $\gamma_{4}$ & $\gamma_{5}$ & $\gamma_{6}$ & $\gamma_{7}$ & $\gamma_{8}$ & $\gamma_{9}$ \\
\hline 1 & $y_{1}(t), \tau=1 \mathrm{~s}$ & 1,54 & 0,91 & 1,04 & 0,85 & 1,11 & $-1,58$ & $-1,41$ & 1,00 & 1,00 & $-5,29$ \\
2 & $y_{1}(t), \tau=1,5 \mathrm{~s}$ & 1,50 & 0,92 & 1,00 & 0,83 & 1,11 & $-1,49$ & $-1,34$ & 0,67 & 0,67 & $-5,28$ \\
3 & $y_{2}(t), \mathrm{n}=1$ & 1,46 & 0,91 & 1,06 & 0,81 & 1,13 & $-11,47$ & $-7,81$ & 0,81 & 0,63 & $-12,99$ \\
4 & $y_{3}(t), \tau=0,3 \mathrm{~s}$ & 1,59 & 0,87 & 2,26 & 0,81 & 1,13 & $-37,58$ & $-9,17$ & 1,43 & 1,04 & $-49,15$ \\
5 & $y_{3}(t), \tau=0,5 \mathrm{~s}$ & 1,48 & 0,87 & 1,82 & 0,88 & 1,18 & $-8,80$ & $-5,88$ & 0,87 & 6,49 & $-18,87$ \\
6 & $y_{4}(t), \tau=0,3 \mathrm{~s}$ & 1,22 & 0,90 & 2,19 & 0,84 & 1,16 & $-32,05$ & $-8,43$ & 1,94 & 1,07 & $-43,44$ \\
7 & $y_{4}(t), \tau=0,5 \mathrm{~s}$ & 1,20 & 1,00 & 1,11 & 0,83 & 1,17 & $-6,20$ & $-4,60$ & 1,22 & 0,69 & $-14,08$ \\
8 & $y_{5}(t), \mathrm{n}=1, \tau=0,15 \mathrm{~s}$ & 1,59 & 0,87 & 2,26 & 0,81 & 1,13 & $-37,58$ & $-9,17$ & 1,43 & 1,04 & $-49,15$ \\
9 & $y_{5}(t), \mathrm{n}=3, \tau=0,15 \mathrm{~s}$ & 1,77 & 0,85 & 1,39 & 0,89 & 1,09 & $-25,77$ & $-10,44$ & 1,15 & 0,81 & $-34,63$ \\
10 & $y_{5}(t), \mathrm{n}=5, \tau=0,15 \mathrm{~s}$ & 1,81 & 0,84 & 1,08 & 0,93 & 1,07 & $-17,01$ & $-10,40$ & 1,02 & 0,70 & $-26,63$ \\
11 & $y_{5}(t), \mathrm{n}=1, \tau=0,1 \mathrm{~s}$ & 1,59 & 0,86 & 3,28 & 0,85 & 1,15 & $-96,77$ & $-8,86$ & 2,14 & 1,57 & $-108,3$ \\
12 & $y_{5}(t), \mathrm{n}=3, \tau=0,1 \mathrm{~s}$ & 1,75 & 0,82 & 2,21 & 0,90 & 1,10 & $-81,50$ & $-10,37$ & 1,73 & 1,22 & $-92,79$ \\
13 & $y_{5}(t), \mathrm{n}=5, \tau=0,1 \mathrm{~s}$ & 1,83 & 0,85 & 1,69 & 0,93 & 1,06 & $-68,61$ & $-11,34$ & 1,53 & 1,04 & $-77,97$ \\
14 & $y_{5}(t), \mathrm{n}=7, \tau=0,1 \mathrm{~s}$ & 1,85 & 0,87 & 1,42 & 0,95 & 1,06 & $-57,50$ & $-11,65$ & 1,40 & 0,93 & $-67,70$ \\
\hline & & & & & & & & & & \\
& & & & & & & & & & &
\end{tabular}

\section{Quality Functional of Wavelet-Basis Choice for Analysis of UWB Signals}

It is proposed to apply the quality functional in the analysis of the UWB signals allowing optimal choice of basic wavelets functions in such a form

$$
J(\psi, y)=\sum_{k=0}^{N}\left|\frac{\gamma_{k}^{\psi}-\gamma_{k}^{y}}{\gamma_{k}^{y}}\right|^{2},
$$

where $\psi(t)$ is wavelet function, $y(t)$ is analyzed signal, $\gamma_{k}^{\psi}$ and $\gamma_{k}^{y}$ are their physical characteristics, and $N$ is quantity of compared parameters. Here, $\gamma_{0}=\mu$ and $N=4$. Below, in Table 3, the values of quality functional of model UWB signal processing for the Kravchenko $\left\{\widehat{h_{2}(t)}\right\}$ and Meyer wavelets are presented. 
Table 3. The values of quality functional of model UWB signal processing for the Kravchenko $\left\{\widetilde{h_{2}(t)}\right\}$ and Meyer wavelets

\begin{tabular}{|c|c|c|c|c|c|c|c|c|c|c|c|c|c|c|}
\hline $\begin{array}{l}\text { No. of } \\
\text { UWB signals } \\
\text { realization }\end{array}$ & 1 & 2 & 3 & 4 & 5 & 6 & 7 & 8 & 9 & 10 & 11 & 12 & 13 & 14 \\
\hline 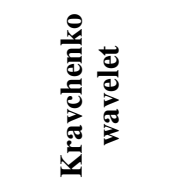 & $\begin{array}{l}\hat{\text { a }} \\
\hat{2}\end{array}$ & જू & $\hat{\mathrm{N}}$ & 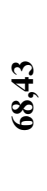 & $\begin{array}{l}n \\
\text { on } \\
\text { ô }\end{array}$ & $\stackrel{F}{\stackrel{F}{F}}$ & $\begin{array}{l}\text { Oे } \\
\text { ป }\end{array}$ & $\begin{array}{l}\text { fo } \\
\text { of }\end{array}$ & है & लै & $\begin{array}{l}\infty \\
\infty \\
\infty \\
\infty\end{array}$ & $=$ & ڤे & $\begin{array}{l}\text { के } \\
\text { ஜ̂ }\end{array}$ \\
\hline 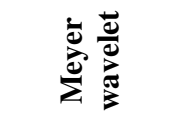 & $\frac{n}{n}$ & $\begin{array}{l}\hat{\sigma} \\
i \\
m\end{array}$ & $\begin{array}{l}n \\
n \\
n\end{array}$ & 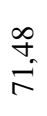 & $\begin{array}{l}\infty \\
\text { N̂ } \\
\text { ñ }\end{array}$ & $\begin{array}{l}\text { ָิ } \\
\text { in }\end{array}$ & $\begin{array}{l}8 \\
\stackrel{8}{0}\end{array}$ & $\stackrel{\infty}{\stackrel{\infty}{+}}$ & $\stackrel{0}{\stackrel{0}{=}}$ & 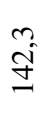 & $\begin{array}{l}\text { 寸 } \\
\text { a }\end{array}$ & 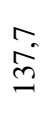 & $\stackrel{0}{n}$ & 6ิ \\
\hline
\end{tabular}

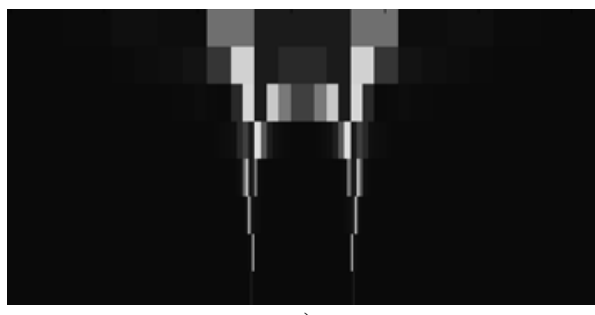

a)

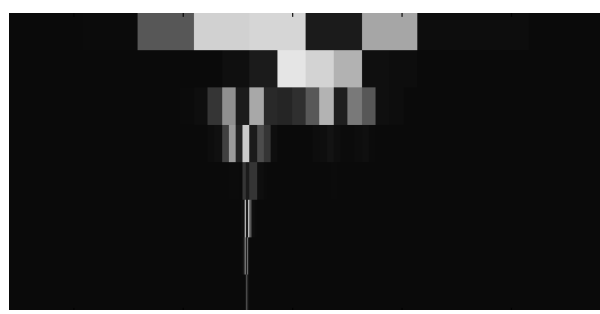

c)

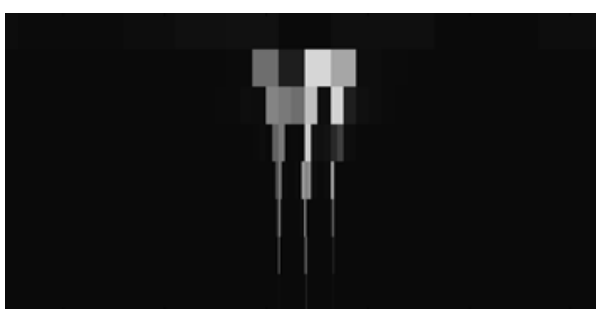

b)

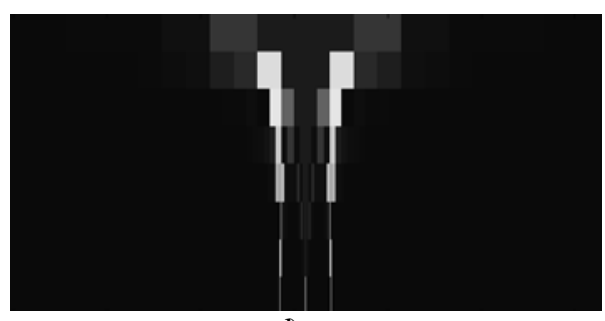

d)

Fig. 2. Discrete wavelet coefficients of $1^{\text {st }}(a), 3^{\text {th }}(b), 4^{\text {th }}(c)$, and $7^{\text {th }}(d)$ UWB signals realization for the Kravchenko $\left\{\widetilde{h_{2}(t)}\right\}$ wavelet

\section{Conclusions}

In this article, the application of the orthogonal Kravchenko wavelets $\left\{\widetilde{h_{a}(t)}\right\}$ for the digital UWB signal processing is proposed and justified. A number of the test signals and their wavelet transform examples are presented. A numerical experiment carried out and also an analysis of physical results have shown the advantages of novel wave- 
let class in its applications for areas of remote sensing, radar, computer vision, radio physics, etc.

Acknowledgments. The investigations were supported by grant NSh-5708.2008.9 "The new methods in some problems of acoustooptics, radio physics and medicine on the basis of atomic functions, wavelets and fractals".

\section{References}

1. Soumekh, M.: Synthetic Aperture Radar Signal Processing with MATLAB Algorithms. John Wiley \& Sons, Inc., NY (1999)

2. Cumming, I.G., Wong, F.H.: Digital Processing of Synthetic Aperture Radar Data: Algorithms and Implementation. Artech house, Inc. Norwood (2005)

3. Ghavami, M., Michael, L.B., Kohno, R.: Ultra Wideband Signals and Systems in Communication Engineering. John Wiley \& Sons, Ltd., Chichester (2004)

4. Arslan, H., Chen, Z.N., Benedetto, M.G.: Ultra Wideband Wireless Communication. John Wiley \& Sons, Ltd., Chichester (2006)

5. Kravchenko, V.F.: Lectures on the Theory of Atomic Functions and Their Some Applications. Publishing House Radio Engineering, Moscow (2003)

6. Kravchenko, V.F., Rvachov, V.L.: Boolean Algebra, Atomic Functions and Wavelets in Physical Applications. Fizmatlit, Moscow (2006)

7. Kravchenko, V.F. (ed.): Digital Signal and Image Processing in Radio Physical Applications. Fizmatlit, Moscow (2007)

8. Kravchenko, V.F., Labun'ko, O.S., Lerer, A.M., Sinyavsky, G.P.: Computing Methods in the Modern Radio Physics. In: Kravchenko, V.F. (ed.) Fizmatlit, Moscow (2009)

9. Meyer, Y.: Wavelets and Operators. Cambridge University Press, Cambridge (1992)

10. Daubechies, I.: Ten Lectures on Wavelets. Society for Industrial \& Applied Mathematics, U.S. (1992)

11. Mallat, S.G.: A Wavelet Tour of Signal Processing. Academic Press, NY (1998)

12. Kravchenko, V.F., Churikov, D.V.: Digital Signal and Image Processing on Basis of Orthogonal Kravchenko Wavelets. In: Proc. Int. Conference "DAYS on DIFFRACTION", May 26-29, pp. 53-54. St.Petersburg, Russia (2009)

13. Kravchenko, V.F., Churikov, D.V.: Atomic Functions $h_{a}(x)$ and New Orthogonal Wavelets on Their Basis. Successes of Modern Radio Electronics 6, 67-88 (2008)

14. Kravchenko, V.F., Churikov, D.V.: A New Class of Orthogonal Kravchenko WA-system Functions $\left\{h_{a}(t)\right\}$. Telecommunications and Radio Engineering 68(8), 649-666 (2009)

15. Gulyaev, Y.V., Kravchenko, V.F., Pustovoit, V.I.: A New Class WA-systems of Kravchenko-Rvachev Functions. Doklady Mathematics 75(2), 325-332 (2007)

16. Kravchenko, V.F., Churikov, D.V.: A New Class of Orthogonal Kravchenko Wavelets. In: Proceedings of International Conference RVK 2008 and MMWP 2008, Växjö, Sweden, June 9-13, pp. 39-43 (2008)

17. Gomeztagle, F., Kravchenko, V.F., Ponomaryov, V.I.: Super-resolution Method Based on Wavelet Atomic Functions in Images and Video Sequences. Telecommunications and Radio Engineering 68(9), 747-761 (2009) 